\title{
Theoretical Modelling and Effectiveness Study of Slotted Stand-Off Layer Damping Treatment for Rail Vibration and Noise Control
}

\author{
Caiyou Zhao ${ }^{1,2}$ and Ping Wang ${ }^{1,2}$ \\ ${ }^{1}$ Key Laboratory of High-Speed Railway Engineering, Ministry of Education, Southwest Jiaotong University, Chengdu 610031, China \\ ${ }^{2}$ School of Civil Engineering, Southwest Jiaotong University, Chengdu 610031, China \\ Correspondence should be addressed to Caiyou Zhao; zcy848279@163.com
}

Received 9 August 2014; Revised 22 November 2014; Accepted 25 November 2014

Academic Editor: Tony Murmu

Copyright (c) 2015 C. Zhao and P. Wang. This is an open access article distributed under the Creative Commons Attribution License, which permits unrestricted use, distribution, and reproduction in any medium, provided the original work is properly cited.

\begin{abstract}
A promising means of reducing railway noise is to increase the damping of the rail, which decreases the vibration of the rail to reduce noise. To achieve this goal, a slotted stand-off layer damping treatment has been developed, and a compound track model with this treatment is developed for investigating the effectiveness of this treatment in terms of the vibration reduction. Through the dynamic analysis of the track undergoing the slotted stand-off layer damping treatment, some guidelines are proposed on the selection of materials and structure parameters for this treatment. In addition, the prototype of the optimal slotted stand-off layer damping treatment has been built and tested in the laboratory. It is found that the slotted stand-off damping treatment shows significant effects in decreasing the amplitude of the accelerance of the rail and a significant reduction of sound emission reflected as the radiation sound pressure level decreases by 8.2 and $9.4 \mathrm{~dB}$ at vertical excitation and lateral excitation, respectively, in the frequency range of $0-4000 \mathrm{~Hz}$.
\end{abstract}

\section{Introduction}

With the development of the high-speed railway and urban rail transit, railway noise has become a growing public concern in the world today. There are plenty of noise sources associated with the railway. However, in general, railway noise is composed mainly of three types: rolling noise, traction noise, and aerodynamic noise [1]. Figure 1 presents the predominant noise sources in the total noise level depending on train speed, of which rolling noise is the greatest contributor to the overall noise level when the train is running at a speed of less than $300 \mathrm{~km} / \mathrm{h}[2]$.

Extensive work has been conducted to understand the mechanism of railway rolling noise generation [3-6]. The vibration of the wheel, rail, and sleeper caused by surface roughness of the rail and train wheel contributes to the overall spectrum of noise. Figure 2 shows, over the whole frequency range, the contribution of the three elements. It is an example of the calculations by Thompson with the TWINS software, which have been validated in extensive field measurements
$[7,8]$. Amongst them, the rail is a dominant source in the frequency region of $450-2000 \mathrm{~Hz}$, and it generates a larger contribution to the total noise above $2000 \mathrm{~Hz}$. This fact means that rail radiation is one of the significant sources of rolling noise.

Numerous measurements have been taken to mitigate the acoustic emission of rails; however, the application of dampers to the rail, in the form of dynamic absorbers or superficial damping layers, is theoretically and practically the most effective. A dynamic absorber can be designed to affect only some specific frequencies so that their damping effect is concentrated within the frequency ranges of interest. Many experts have paid much attention to the application of dynamic absorbers in rails. Maes and Sol presented a double tuned rail damper, which is used to reduce the first two vertical "pined-pined" vibrations of the rail. The damper is mounted between two sleepers on the rail. Measurements at a test track of the French railway company showed that the wave decay rate of rail vibration can be effectively increased by the rail damper [9]. Ho et al. developed a 


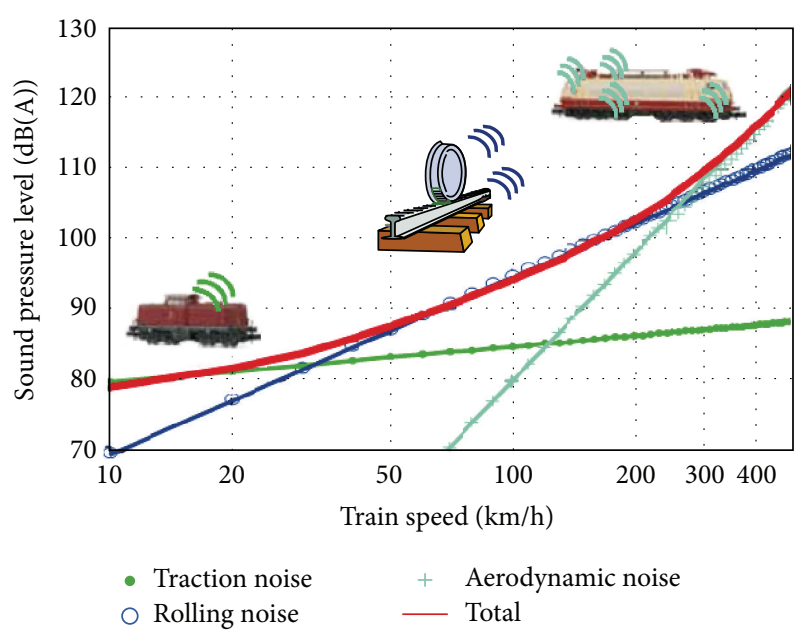

FIgURE 1: Relative strength and speed dependence of sources of railway noise [2].

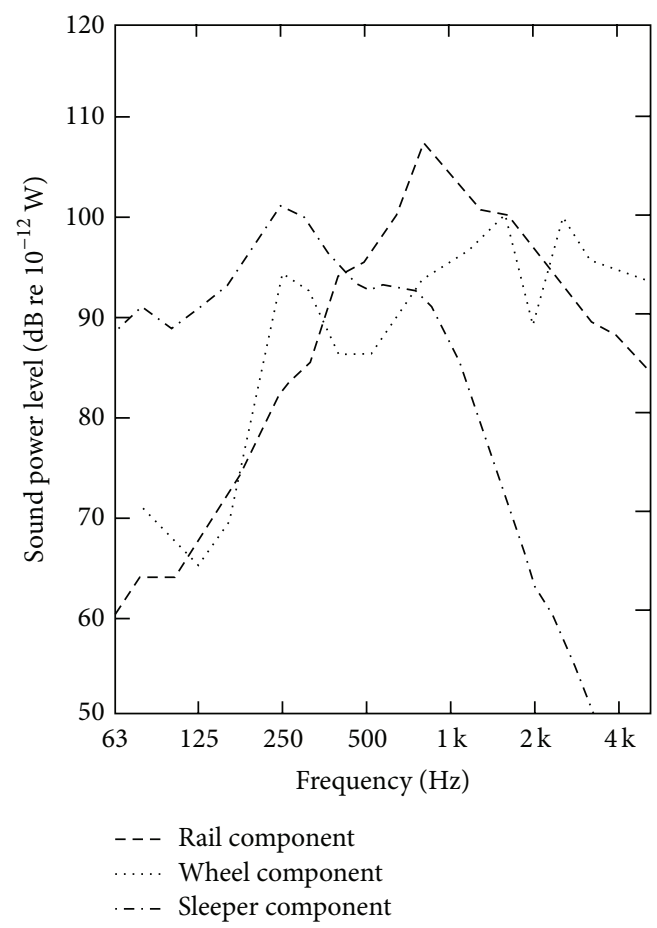

Figure 2: Noise emissions of the track $[7,8]$.

new type of tuned mass damper which comprises multiple masses oscillating along the shear direction of resilient layers forming a multiple-mass-spring system and it can reduce the rolling noise of track components by $5 \mathrm{~dB}$ in field tests [10]. Ltourneaux et al. studied another rail damper, each unit of which was shorter but wider than Thompson's damper. A reduction of $3 \mathrm{~dB}$ in overall noise was found in tests on a running line with pads of medium stiffness, approximately $350 \mathrm{kN} / \mathrm{mm}$ [11]. Asmussen et al. produced a rail damper that consisted of steel masses and elastomer-based materials. The elastomer was used to absorb energy through internal friction as the steel elements vibrate in response to vibrations in the rail. On a test track, a noise reduction up to $4 \mathrm{~dB}$, due to the increased damping of the rail, was measured [12].

While dynamic absorbers affect only some specific frequencies, superficial damping layers can be designed to act on the entire frequency range, depending on the viscoelastic property of the damping material. Similarly, many studies have been conducted in the past to investigate noise abatement characteristics of superficial damping layers and their applications on rails. Wei et al. developed a compound damping board, a complex of high damping material and restrained board. An experimental study on the dynamic behaviour of rail attached to the compound damping board has been presented. The results of field tests prove that the compound damping board can reduce the lateral vibration level of rails by 6-7 $\mathrm{dB}$ [13]. Li et al. built a constrained damping layer that was made of a damping layer ( $2 \mathrm{~cm}$ thick) and an aluminium constrained layer ( $3 \mathrm{~cm}$ thick). Research results showed that these damped rails had a good effect on vibration reduction in a wide frequency range of $0-5000 \mathrm{~Hz}$ [14]. Xue Jun offered a labyrinth constrained damping layer that consisted of three parts: a connection layer, a damping layer, and a constrained layer. The field experiment results showed that the labyrinth constrained damping layer can reduce rail noise by up to $7 \mathrm{~dB}$ [15]. In general, these studies have focused on the application of traditional superficial free-damping layers or superficial constrained damping layers to dissipate rail vibration energy, reducing the acoustic emission of rails. However, little effort has been devoted to the application of more efficient damping treatment measurement in rails.

The research presented herein constitutes an exploratory study of the slotted stand-off layer damping treatment for rail vibration and noise control using both theoretical analysis and laboratory tests. First, the working principle of the slotted stand-off layer damping treatment is discussed. Then, a compound track model with this treatment is developed for investigating the effectiveness of this treatment in terms of vibration reduction. Additionally, some guidelines are provided on the selection of materials and structure parameters by the model for this treatment. Finally, the optimal slotted stand-off layer damping treatment is used to make a full scale sample. The vibration and acoustic reduction effect is measured in the laboratory.

\section{Working Principle of the Slotted Stand-Off Layer Damping Treatment}

Applying a surface damping treatment to a stiff beam, plate, shell, and other thin-walled structures can reduce the amplitude of a vibration [16]. When the damping treatment is applied as a single-layer coating, sometimes known as a free-layer damping, energy is dissipated as a result of the extension and compression of the damping material while the base structure bends during vibration. A significant increase in energy dissipation can be achieved by attaching a stiff layer (or constraining layer) on top of the viscoelastic layer, as shown in Figure 3. This dissipation occurs because large shear strains are generated in the damping material when the system bends during vibration. Adding a stand-off layer 


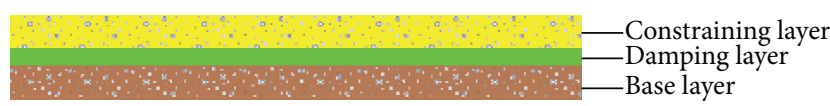

(a) Before impact

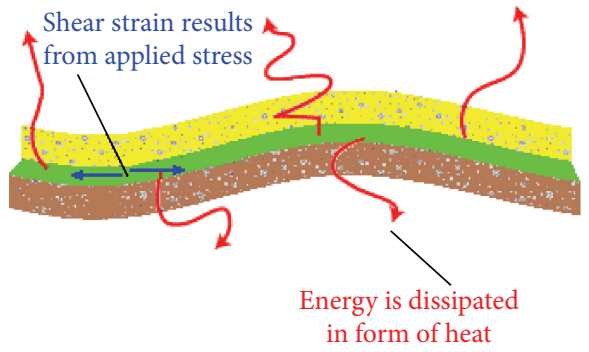

(b) Being deformed during vibration

FIGURE 3: Working principle of a constraining damping treatment system.

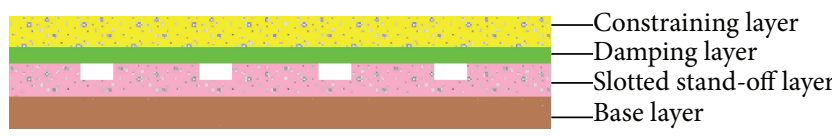

(a) Before impact

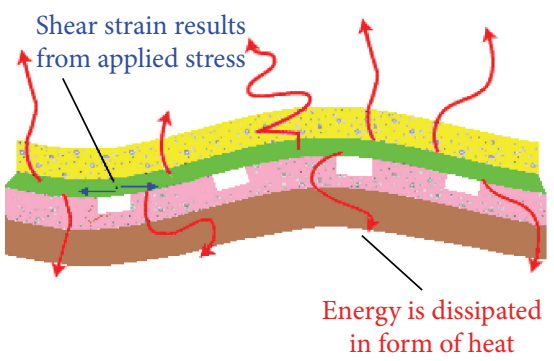

(b) Being deformed during vibration

FIGURE 4: Working principle of a slotted stand-off layer damping treatment.

to the constraining damping treatment between the base layer and the damping layer could further increase the shear angle of the damping layer. This damping treatment has been termed passive stand-off layer damping treatment. However, for the effective operation of passive stand-off layer damping treatment, the stand-off layer must have high shear stiffness and, at the same time, must not significantly affect the bending stiffness of the composite structure. Based on this principle, in one variation of passive stand-off layer damping treatment, slots are introduced into the stand-off layer. Slotted stand-off layer damping treatment, as shown in Figure 4, has been thought to have an important advantage over continuous passive stand-off layer damping treatment [17].

In general, the stand-off layer has no internal damping properties. Therefore, the damping ratio $\eta$ is used to assess the vibration reduction effect of the stand-off layer damping treatment as the traditional constraining damping treatment. The damping ratio $\eta$ can be defined, according to the "strain energy method," as the ratio between the energy dissipated by

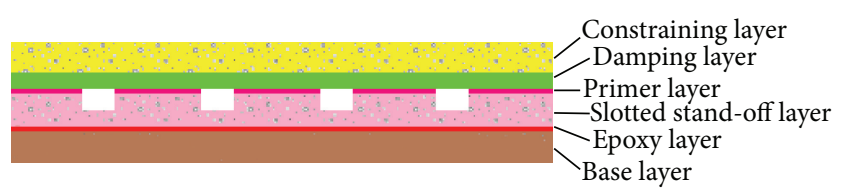

FIGURE 5: Schematic diagram of slotted stand-off layer damping treatment.

the damping layer in each strain cycle and that stored in the system:

$$
\eta=\frac{D_{0}}{2 \pi W_{0}}
$$

where $D_{0}$ is the dissipated energy in the damping layer and $W_{0}$ is the stored energy of the system in a strain cycle.

As for $D_{0}$,

$$
D_{0}=\pi \cdot G^{\prime \prime}(\omega) \cdot V \cdot \gamma^{2},
$$

where $\gamma$ is the shear strains of the damping layer, $G^{\prime \prime}(\omega)$ is the loss modulus of the damping material, and $V$ is the volume of the damping layer. The addition of a slotted stand-off layer only increases shear strains, thereby reducing the vibration of the base layer more effectively.

\section{Optimisation Analysis of Slotted Stand-Off Layer Damping Treatment}

Some studies have been conducted in the past to investigate the application of stand-off layer damping treatment in industrial fields and the corresponding calculation of their damping ratios. Generally, the theoretical solution for the loss factor calculation is unfortunately available only for simple geometry structures. More recently, an alternative approach, based on numerical finite element calculations, allowed the consideration of a different component shape, as in the case of a rail. Furthermore, in the slotted stand-off layer damping treatment system, the bonding layers used to connect these layers are found to have a measurable and significant effect on the response of the structure. Therefore, the finite element model presented here includes an epoxy layer between the base layer and the slotted stand-off layer and a contact cement layer between the slotted stand-off layer and the damping layer. The six-layered model incorporating a primer layer and an epoxy layer, as shown in Figure 5, proposed by Yellin et al. [18], is adopted in this study to model slotted stand-off layer damping treatment.

3.1. Numerical Procedure for the Damping Ratio Calculation. In the present work, an approach based on a steady-state harmonic analysis in the frequency range of interest has been adopted. This approach directly integrates the equations of motion of the system. Thus, all of the vibration modes involved at any excitation frequency will be taken into consideration. This approach, therefore, simulates the actual behaviour of the component. To this end, a postprocessing 
procedure for the ANSYS commercial code has been developed and can be generally applied to any component shape and material. In particular, the dependence of the elastic and viscous properties of the damping layer material on frequency and temperature will be taken into account to define these properties directly as supplied by the producer. The results calculated in this way are very flexible.

The specific procedure calculates the damping ratio $\eta$ in the discretized form.

(a) The dissipated energy $D_{0}$ mainly derives from the shear deformations of the damping layer:

$$
D_{0}=\sum_{i}\left(D_{0}\right)_{i}=\sum_{i=1} 2 \pi W_{\text {shear }_{i}}^{\prime \prime}
$$

whereby

$$
W_{\text {shear }_{i}}^{\prime \prime}=\iiint \frac{G^{\prime \prime}(\omega)}{2} \sum_{j=1}^{2} \gamma_{0 j}^{2} d x d y d z
$$

so,

$$
D_{0}=\pi \cdot G^{\prime \prime}(\omega) \sum_{i}\left[V_{i} \sum_{j=1}^{2}\left(\gamma_{0 j}^{2}\right)_{i}\right] \text {. }
$$

(b) The stored energy $W_{0}$ mainly derives from two parts, namely, the stored energy from the damping layer generated by shear deformations and the stored energy from the other five layers generated by elastic deformations:

$$
W_{0}=\sum_{i}\left(W_{0}\right)_{i}=\sum_{i}\left(W_{S}+W_{V}+W_{\text {shear }}^{\prime}\right)
$$

where $W_{S}, W_{V}$, and $W_{\text {shear }}^{\prime}$ denote the elastic deformation energy.

For the $i$ th element $(i=1 \ldots n)$,

$$
\begin{gathered}
W_{i}=\frac{1}{2}\left(\sigma_{1} \varepsilon_{1}+\sigma_{2} \varepsilon_{2}+\sigma_{3} \varepsilon_{3}\right) \\
=\frac{1}{2 E}\left[\sigma_{1}^{2}+\sigma_{2}^{2}+\sigma_{3}^{2}-2 v\left(\sigma_{1} \varepsilon_{1}+\sigma_{2} \varepsilon_{2}+\sigma_{3} \varepsilon_{3}\right)\right] \\
W_{V i}=\frac{1}{2} \cdot \frac{\sigma_{1}+\sigma_{2}+\sigma_{3}}{3}\left(\varepsilon_{1}+\varepsilon_{2}+\varepsilon_{3}\right) \\
=\frac{1-2 v}{6 E}\left(\sigma_{1}+\sigma_{2}+\sigma_{3}\right)^{2} \\
=\frac{3(1-2 v)}{2 E} \sigma_{\text {oct }}^{2},
\end{gathered}
$$

$$
\begin{aligned}
& W_{S i}= W_{i}-W_{V i} \\
&= \frac{1}{2 E}\left[\sigma_{1}^{2}+\sigma_{2}^{2}+\sigma_{3}^{2}-2 v\left(\sigma_{1} \sigma_{2}+\sigma_{2} \sigma_{3}+\sigma_{1} \sigma_{3}\right)\right. \\
&\left.\quad \quad-\frac{1-2 v}{3}\left(\sigma_{1}+\sigma_{2}+\sigma_{3}\right)^{2}\right] \\
&= \frac{1}{12 G}\left[\left(\sigma_{1}-\sigma_{2}\right)^{2}+\left(\sigma_{2}-\sigma_{3}\right)^{2}+\left(\sigma_{1}-\sigma_{3}\right)^{2}\right] \\
&= \frac{1}{6 G} \sigma_{\text {Mises }}^{2} \\
&= \frac{1+v}{3 E} \sigma_{\text {Mises }}^{2}, \\
& \quad W_{\text {shear }}^{\prime}=\frac{G^{\prime}(\omega)}{2} \sum_{j=1}^{2} \gamma_{0 j}^{2} .
\end{aligned}
$$

Substituting (7) into (6) gives

$$
\begin{gathered}
W_{0}=\sum_{i} V_{i}\left[\frac{1+\nu}{3 E} \sigma_{\text {Mises }_{i}}^{2}+\frac{3(1-2 \nu)}{2 E} \sigma_{\text {oct }_{i}}^{2}\right. \\
\left.+\frac{G^{\prime}(\omega)}{2} \sum_{j=1}^{2}\left(\gamma_{0 j}^{2}\right)_{i}\right],
\end{gathered}
$$

where $V_{i}$ is the volume of the $i$ th element, $\gamma_{0 j}$ is the $j$ th shear deformation of the $i$ th element, $\sigma_{\text {Mises }_{i}}$ is the von Mises stress of the $i$ th element, $\sigma_{\text {oct }_{i}}$ is the octahedral stress of the $i$ th element, $G^{\prime}$ is the storage shear modulus, $G^{\prime \prime}$ is the loss shear modulus, $E$ is Young's modulus of elasticity, and $v$ is the Poisson ratio.

The damping ratio $\eta$ can be obtained by substituting (5) and (8) into (1)

3.2. Choice of the Material and Configuration of the Slotted Stand-Off Layer Damping Treatment. Several commercial damping materials were examined in this project. One of these, VER-IPN, was chosen because of its high loss-factor values in the temperature and frequency ranges of interest, as depicted in Figure 6. The slotted stand-off layer was made from Dyad606 with high shear strength. Its density and Young's modulus of elasticity were $1200 \mathrm{~kg} / \mathrm{m}^{3}$ and $500 \mathrm{MPa}$, respectively, as tested. Although the epoxy layer and primer layer were relatively thin, they should be as stiff as possible; therefore, a strong adhesive that has good synthetic properties was developed. The adhesive had a tensile modulus of approximately $1.65 \mathrm{GPa}$. The material used for the constraining layer can be either Al-2A14, with an elastic modulus of $E=$ $73.1 \mathrm{GPa}$, or steel-3Cr13Mo, with an elastic modulus of $E=$ $206 \mathrm{GPa}$.

However, it is not easy to choose the best arrangement for slotted stand-off damping treatment. The rail waist and the rail foot are the weakest zones of the rail; therefore, such parts appear to be the most feasible in terms of application 


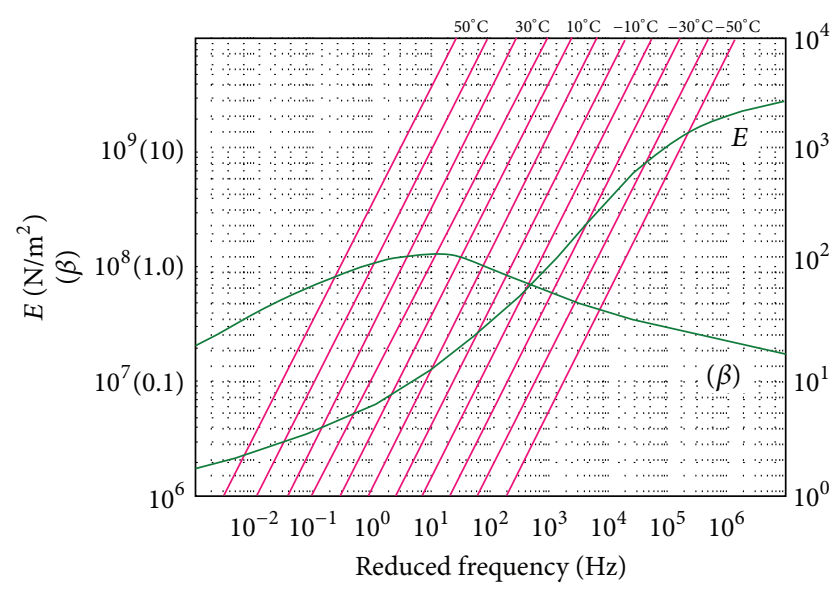

FIGURE 6: Damping VER-IPN property specification.

and the most promising in terms of effectiveness. However, this solution shows difficulties in shaping the damping layer to match the rail profile. A $60 \mathrm{~kg} / \mathrm{m}$ rail, which is the most widely used in China, is studied in this paper. The basic idea is to apply a slotted stand-off damping treatment to specified zones of a widely used rail, without additional machining operations on the rail itself. Several solutions were initially proposed and examined, taking the following design requirements into consideration: damping efficiency, safety, and reliability under working conditions (in particular mechanical resistance, weathering resistance, moisture and solvent resistance, and thermal and fire resistance), ease of manufacturing and application, weight, and cost. Finally, the slotted stand-off layer damping treatment is arranged according to the special structure of the rail, as depicted in Figure 7, consisting of four major parts: an inverted T-shaped constraining layer of uniform thickness (3), connected to the rail with a slotted stand-off layer with uniform thickness (1) and sandwiched between the slotted stand-off layer and the constraining layer with a slot with a thickness-variable damping layer (2); the rail (4) is surrounded by stand-off layer. The strong adhesive is applied to each layer.

The numerical procedure described above was used to examine the effectiveness of different configurations. The modifiable structural size of the slotted stand-off layer damping treatment was defined, as shown in Figure 8. In particular, the calculations were carried out for the following seven cases (also shown in Table 1), where $h_{s 1}$ is the zone thickness of the slotted stand-off layer without slots, $h_{s 2}$ is the slot thickness of the slotted stand-off layer, $t_{s 1}$ is the slot width, $t_{s 2}$ is the zone width of the slotted stand-off layer without slots, $h_{c}$ is the thickness of the constraining layer, $t_{c 1} \sim t_{c 3}$ are the three important structural sizes of the constraining layer, and $\alpha$ is the slope of the constraining layer.

Previous research has shown that the length of a rail mode with twelve spans can yield the whole resonance and antiresonance characteristics of a rail in the frequency range of $0 \sim 5000 \mathrm{~Hz}$ [19]. Thus, the length of the rail mode with twelve spans (with a total length of $7.2 \mathrm{~m}$ ) is applied in the present work. The rails are fastened by the WJ-7 flexible fastening systems every $0.6 \mathrm{~m}$, and the fastening systems are fixed on the prestressed reinforced concrete sleepers III. Moreover, the operating temperature of the damping material is assumed to be $30^{\circ} \mathrm{C}$. To represent realistic working conditions, two forcing actions, one twice as much as the other, are applied simultaneously on the railhead surface in the vertical and lateral directions, as shown in Figure 9; these actions simulate the typical main loads transmitted from the wheel to the rail (11).

The force $F$ is calculated as follows:

$$
\begin{aligned}
& \quad F=\left(1+\alpha_{1}\right) \cdot\left(1+\alpha_{2}\right) \cdot P, \\
& \text { where } \alpha_{1}=0.6 \frac{v}{100}, \quad \alpha_{2}=0 \text {, } \\
& \text { when } v \text { is less than } 120 \mathrm{~km} / \mathrm{h} \text {, }
\end{aligned}
$$

$$
\begin{array}{r}
\alpha_{1}=0.72, \quad \alpha_{2}=0.3 \frac{\Delta v}{100}, \quad \Delta v=v-120, \\
\text { when } v \text { is larger than } 120 \mathrm{~km} / \mathrm{h},
\end{array}
$$

where $v$ is the vehicle speed and $P$ is the axle load of the vehicle.

The axle load $P$ of $15 \mathrm{~T}$ (CRH3 high-speed train) is studied in this paper, and the speed of the train is $300 \mathrm{~km} / \mathrm{h}$. Therefore, the force $F$ is $397,270 \mathrm{~N}$.

\section{Results}

Thirteen representative frequencies were chosen from the modal analysis of the track model described above. The damping ratios calculated from the seven different cases are shown in Table 2 . The following can be deduced from Table 2.

(a) The damping ratio $\eta$ of the rail with the slotted standoff layer damping treatment is mostly greater than 0.017 . However, the $\eta$ of the standard rail is only $0.0001 \sim 0.0006$. This result means that the damping ratio $\eta$ of the rail with the slotted stand-off layer damping treatment far outweighs the $\eta$ of the standard rail.

(b) The zone thickness of the slotted stand-off layer without slots imposes different influences on specific eigenmodes, as noted by comparing the results of Cases 1 and 2. The thickness of Case 1 is more effective for the first, third, fifth, sixth, eighth, and thirteen eigenmodes, whereas that of Case 2 is more effective for the other eigenmodes. Furthermore, in view of the great rigidity of the material of the slotted stand-off layer, as mentioned, it is more reasonable to apply the thickness from Case 2.

(c) The structural dimensions of the slot exert a strong influence on the damping ratios, as highlighted by comparing the results of Cases 2 and 3 and Cases 4 and 5: the thicker and wider the slot, the greater the damping ratios in the frequency range of greatest interest. The influence of the zone width of the slotted stand-off layer without the slot is contrary to the structural dimensions of the slot: the wider the zone of the slotted stand-off layer without slots, the smaller the total width of the slot. 


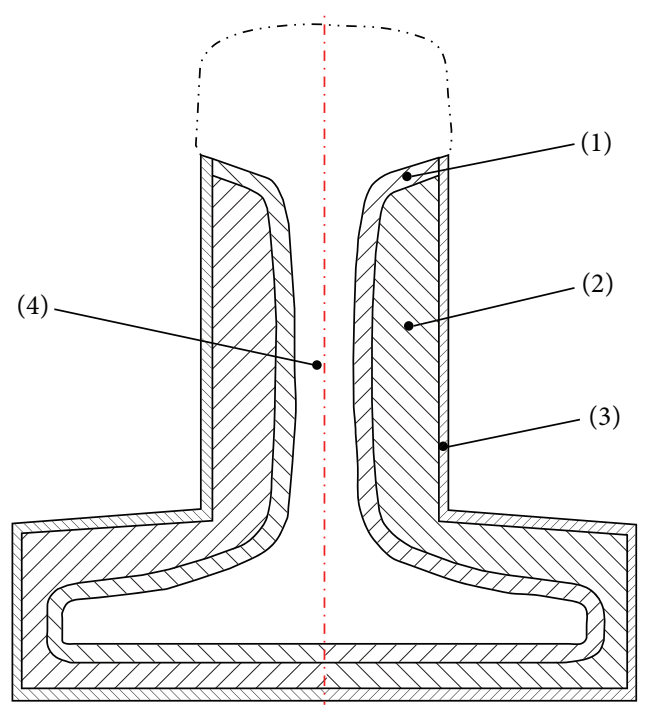

(a) Without slot

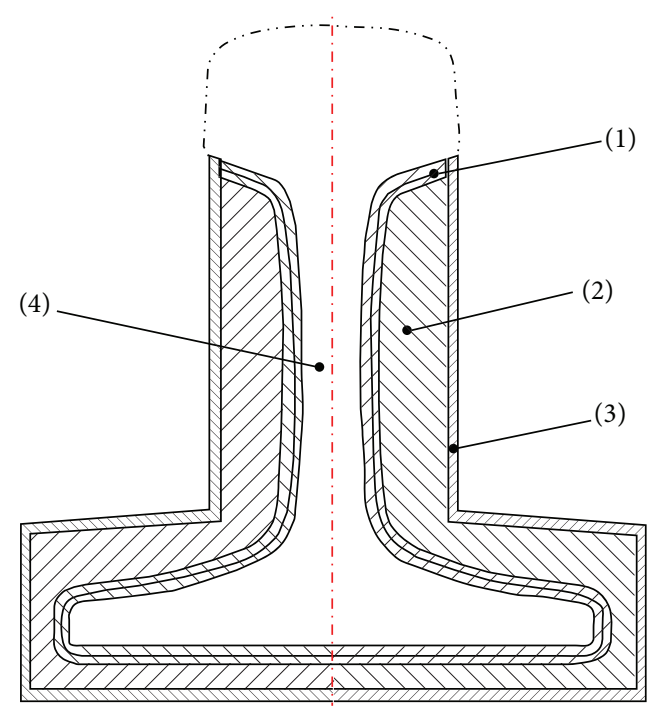

(b) With slot

FIgURE 7: Cross section of the rail with the slotted stand-off layer damping treatment.

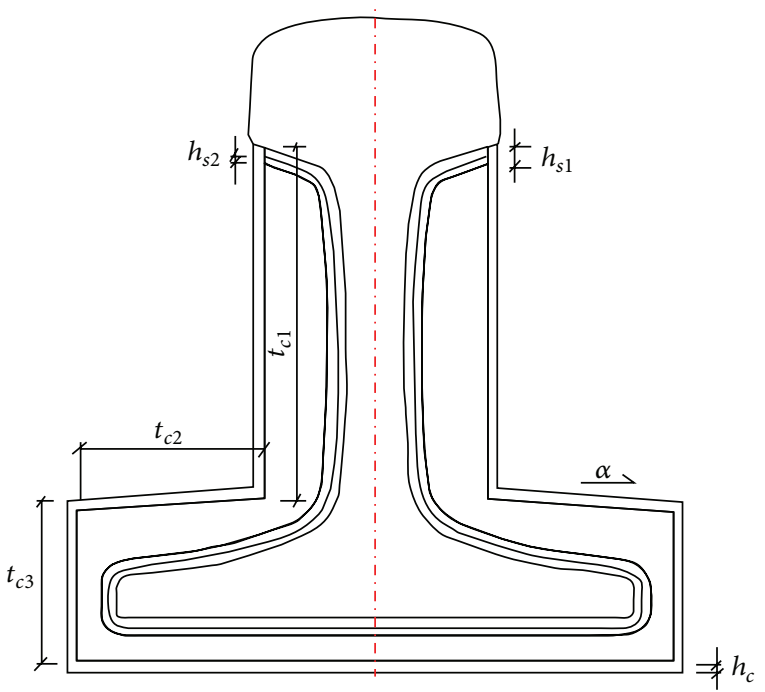

(a) Cross section of the rail with damping treatment

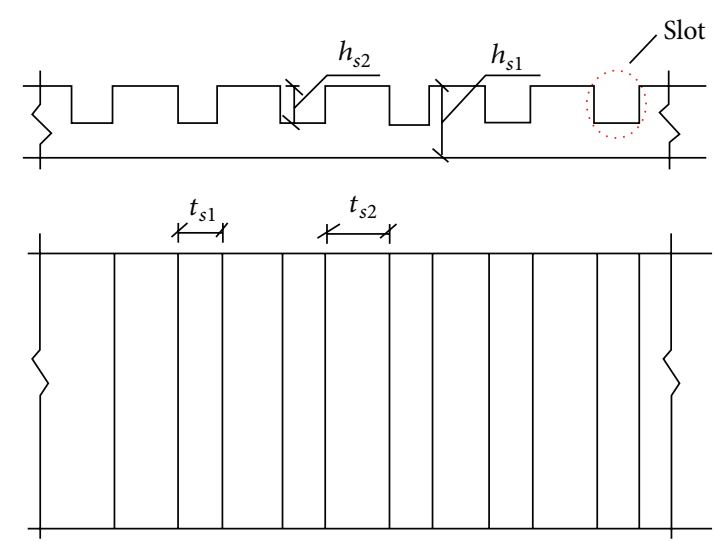

(b) Detailed characteristic of slotted stand-off layer

FIgURE 8: Parameter definitions of the slotted stand-off layer damping treatment.

TABLE 1: Tests of the material and configuration.

\begin{tabular}{|c|c|c|c|c|c|c|c|c|c|c|c|}
\hline Case & $h_{s 1} / \mathrm{mm}$ & $t_{c 1} / \mathrm{mm}$ & $\alpha$ & $t_{c 2} / \mathrm{mm}$ & $t_{c 3} / \mathrm{mm}$ & $h_{c} / \mathrm{mm}$ & $h_{s 2} / \mathrm{mm}$ & $t_{s 2} / \mathrm{mm}$ & $t_{s 1} / \mathrm{mm}$ & $\begin{array}{c}\text { Damping layer } \\
\text { material }\end{array}$ & $\begin{array}{c}\text { Constraining layer } \\
\text { material }\end{array}$ \\
\hline 1 & 8 & 100 & $4 \%$ & 55 & 45 & 3 & 2 & 30 & 20 & VER-IPN & Al-2A14 \\
\hline 2 & 6 & 100 & $4 \%$ & 55 & 45 & 3 & 2 & 30 & 20 & VER-IPN & Al-2A14 \\
\hline 3 & 6 & 100 & $4 \%$ & 55 & 45 & 3 & 3 & 30 & 20 & VER-IPN & Al-2A14 \\
\hline 4 & 6 & 100 & $4 \%$ & 55 & 45 & 3 & 3 & 20 & 20 & VER-IPN & $\mathrm{Al}-2 \mathrm{~A} 14$ \\
\hline 5 & 6 & 100 & $4 \%$ & 55 & 45 & 3 & 3 & 20 & 30 & VER-IPN & $\mathrm{Al}-2 \mathrm{Al} 4$ \\
\hline 6 & 6 & 100 & $4 \%$ & 55 & 45 & 2 & 3 & 20 & 30 & VER-IPN & $\mathrm{Al}-2 \mathrm{~A} 14$ \\
\hline 7 & 6 & 100 & $4 \%$ & 55 & 45 & 2 & 3 & 20 & 30 & VER-IPN & Steel-3Cr13Mo \\
\hline
\end{tabular}


TABLE 2: Damping ratio $\eta$ of the seven cases.

\begin{tabular}{lccccccc}
\hline Frequency & Case 1 & Case 2 & Case 3 & Case 4 & Case 5 & Case 6 & Case 7 \\
\hline 100 & 0.018 & 0.017 & 0.019 & 0.021 & 0.025 & 0.024 & 0.028 \\
200 & 0.022 & 0.023 & 0.024 & 0.026 & 0.031 & 0.031 & 0.035 \\
600 & 0.033 & 0.031 & 0.034 & 0.035 & 0.044 & 0.042 & 0.044 \\
1000 & 0.041 & 0.042 & 0.044 & 0.048 & 0.059 & 0.061 & 0.065 \\
1300 & 0.132 & 0.128 & 0.119 & 0.129 & 0.163 & 0.156 & 0.187 \\
1500 & 0.209 & 0.231 & 0.221 & 0.248 & 0.287 & 0.293 & 0.358 \\
2100 & 0.164 & 0.163 & 0.171 & 0.187 & 0.212 & 0.218 & 0.175 \\
2500 & 0.132 & 0.128 & 0.162 & 0.158 & 0.176 & 0.185 \\
2700 & 0.121 & 0.130 & 0.152 & 0.175 & 0.185 & 0.194 \\
3100 & 0.108 & 0.121 & 0.132 & 0.154 & 0.146 & 0.144 & 0.191 \\
3400 & 0.290 & 0.321 & 0.414 & 0.374 & 0.392 & 0.389 & 0.132 \\
3700 & 0.415 & 0.436 & 0.356 & 0.498 & 0.564 & 0.544 & 0.614 \\
4000 & 0.479 & 0.387 & 0.412 & 0.453 & 0.534 & 0.512 \\
\hline
\end{tabular}

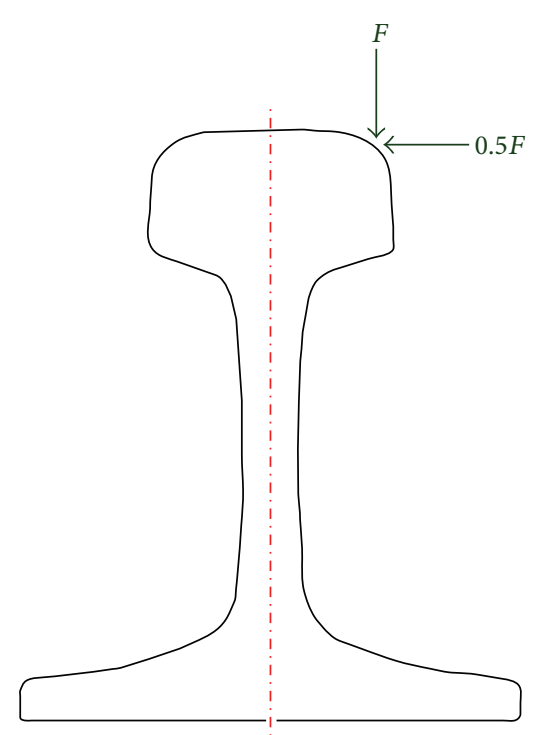

Figure 9: Schematic plot of the forcing actions.

(d) The thickness of the constraining layer has some effect on the damping ratio $\eta$ of the rail with slotted standoff layer damping treatment, as shown by comparing the results of Cases 5 and 6 . Case 5 has damping ratios slightly larger than that of Case 6 in addition to the fourth, sixth, seventh, and ninth eigenmodes. However, a $3 \mathrm{~mm}$ thick aluminium plate or steel plate is more difficult to be machined and shaped than $2 \mathrm{~mm}$ plates. Therefore, it is better to adopt the $2 \mathrm{~mm}$ thick constraining layer. The difference between steel and aluminium constraining layers (Cases 6 and 7) is significant, and the damping ratio of the former is obviously greater than that of the latter.

(e) In sum, to obtain as great of a noise reduction as possible in the frequency range of interest and to manufacture the system as easily as possible, Case 7 is the best choice.

\section{Laboratory Measurements of Vibration and Noise}

Laboratory measurements of accelerance and noise were carried out on the Chinese $60 \mathrm{~kg} / \mathrm{m}$ standard rail with the slotted stand-off layer damping treatment according to Case 7 to test its actual effectiveness compared with that of the standard rail. For the sake of economy and convenience, the length of the standard rail with the slotted stand-off layer damping treatment was $0.6 \mathrm{~m}$, as shown in Figure 10.

The characteristics of this slotted stand-off layer damping treatment are as follows.

(a) It has a total mass of $12 \mathrm{~kg}$. Because the sleeper spacing is assumed to be $0.6 \mathrm{~m}$, the mass per meter of rail length is $20 \mathrm{~kg}$.

(b) It is designed to be fitted on the rail without any modifications made to the rail, to the sleepers, or to the supporting ballast layer.

(c) The adhesive strength of the efficient noise-reduction glue is proven to be effective after 2 million cycles of fatigue testing.

(d) The electrical isolation is maintained.

(e) The radiation of sound by the slotted stand-off layer damping treatment itself is negligible.

5.1. Laboratory Measurements and Analysis of Accelerance. The model used for laboratory measurements and analyses of accelerance of the rail with the slotted stand-off layer damping treatment and standard rail is presented below. The rail grid model was constructed with prestressed reinforced concrete sleepers III, on which there were fastened rails with the slotted stand-off layer damping treatment, or $60 \mathrm{~kg} / \mathrm{m}$ standard rails using WJ-7 flexible fastenings. For the testing of the acceleration admittance of the samples, the method of measuring the response to mechanical shock was used in accordance with ISO 7626-5 [20]. Mechanical shock was stimulated using an impulse hammer in the vertical and lateral direction on the railhead. A part of this hammer is a force 


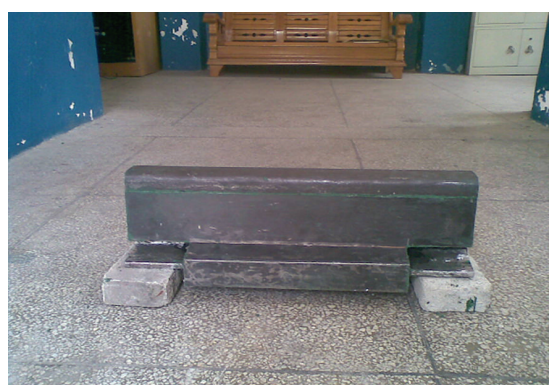

(a) Picture of the rail with slotted stand-off layer damping treatment

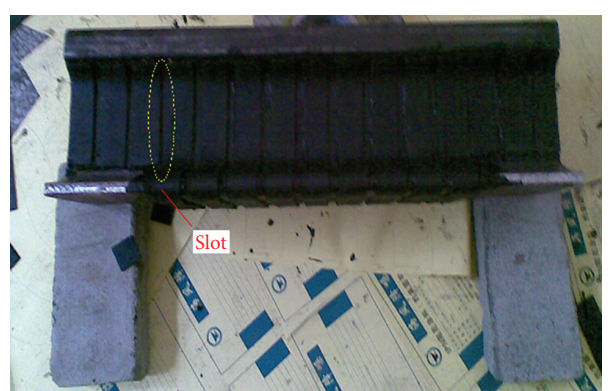

(b) Detailed characteristics of the slotted stand-off layer

FIGURE 10: Schematic plot of rail with the slotted stand-off layer damping treatment.

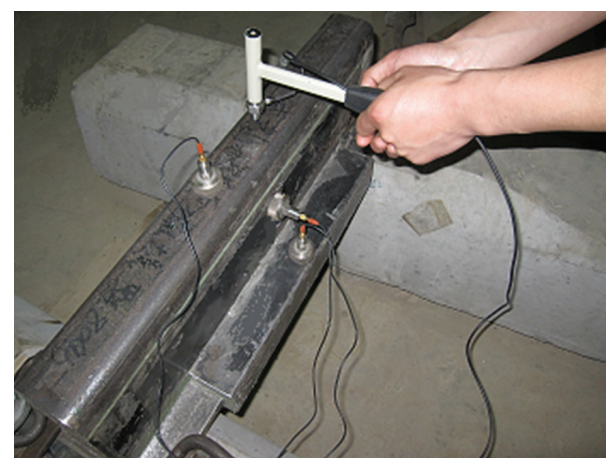

FIgURE 11: Treatment configurations examined.

detector. The response was measured using accelerometers at different points of the rail structure, on the rail head, rail waist, and rail foot of the midpoint of the rail-specimen, as shown in Figure 11.

The measuring system consisted of a DHDAS-5920 PULSE modulator analyser for recording the vibration parameters together with three LC0102 acceleration detectors and a LC1303 shock stimulation hammer. The accelerometers were fastened to the measured construction by means of epoxy resin adhesives.

A comparison of the vertical and lateral accelerance of the rail head between the standard rail and the rail with the slotted stand-off damping treatment is depicted in Figures 12(a) and 12(b), respectively. In Figure 12(a), it is possible to observe a general decrease in the vertical accelerance amplitude of the rail head of the rail with the slotted stand-off damping treatment amplitude in the frequency range of $0-4000 \mathrm{~Hz}$, relative to the case without the slotted stand-off damping treatment, as the maximum acceleration reading decreased from $0.29 \mathrm{~m} \cdot \mathrm{s}^{-2} / \mathrm{N}$ to $0.078 \mathrm{~m} \cdot \mathrm{s}^{-2} / \mathrm{N}$. From Figure 12(b), the lateral accelerance amplitude of the rail with the slotted stand-off damping treatment is approximately $0.0015 \mathrm{~m} \cdot \mathrm{s}^{-2} / \mathrm{N}$ in the low frequency range of $0-200 \mathrm{~Hz}$ and the middle frequency range of $200-1000 \mathrm{~Hz}$. This result means that the lateral vibration of the rail head in a frequency range of $0-2000 \mathrm{~Hz}$ is almost completely damped out by the slotted stand-off damping treatment. Additionally, it can be observed from the figure that compared to the standard rail, the lateral accelerance amplitude of the rail with the slotted stand-off damping treatment decreased from $0.05 \mathrm{~m} \cdot \mathrm{s}^{-2} / \mathrm{N}$ to $0.019 \mathrm{~m} \cdot \mathrm{s}^{-2} / \mathrm{N}$ in the high frequency range of $1000-4000 \mathrm{~Hz}$. Thus, it was possible to obtain significant reductions in the vertical and lateral vibration levels of rail head by taking the slotted stand-off damping treatment approach in the frequency range of $0-4000 \mathrm{~Hz}$.

Similar conclusions are observed in Figures 13 and 14, which present the analysis of the comparison of the accelerance of the rail waist and the rail foot between the standard rail and the rail with the slotted stand-off damping treatment. It can be observed from these figures that the performance of the slotted stand-off damping treatment placed at the surface of the rail for attenuating the vibrations of the rail waist and rail foot is quite effective in the frequency range of $0-4000 \mathrm{~Hz}$.

Based on Figures 12, 13, and 14, it is possible to conclude that the slotted stand-off damping treatment decreases the amplitude of the accelerance of the rail in the frequency range of $0-4000 \mathrm{~Hz}$, especially in the middle frequency range of $200-1000 \mathrm{~Hz}$ and the high frequency range of $1000-4000 \mathrm{~Hz}$.

5.2. Laboratory Measurements and Analysis of Noise. The measurement procedure was conducted according to ISO 3744 for the source sound power emission calculation in freefield conditions by sound pressure measurements [21]. In this case, the sound power, which depends on the exciting force, was normalised by this force. The acoustic measurements were carried out in a semianechoic room according to the engineering method of ISO 3744 in the frequency range of $0-4000 \mathrm{~Hz}$.

The model and its installation are the same as those applied in Section 5.1. The exciting force is an impulse caused by the falling weight $(250 \mathrm{~g})$. The vertical falling location is fixed over the midpoint of the rail-specimen along its length, $40 \mathrm{~cm}$ away from the rail head in the vertical direction and $3.5 \mathrm{~cm}$ away from the rail centreline in the lateral direction. Meanwhile, the lateral falling location is fixed over the midpoint of the rail-specimen along its length, $22 \mathrm{~cm}$ away from the rail head in the vertical direction and $45 \mathrm{~cm}$ away from the rail centreline in the lateral direction, as shown in Figure 15. The measurement was made using a microphone mounted on the midpoint of the rail-specimen along its 


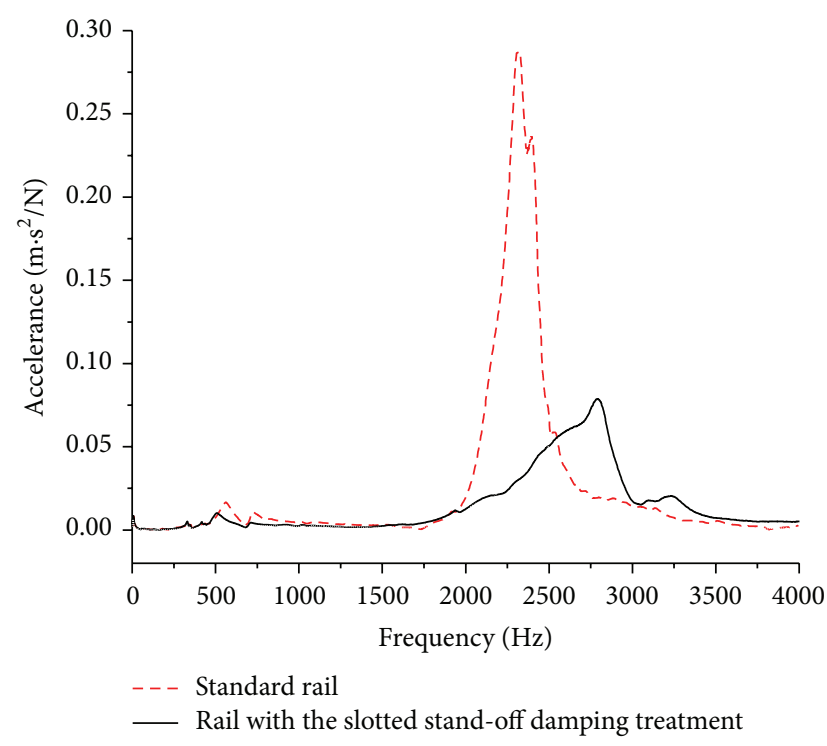

(a) Vertical accelerance of the rail head

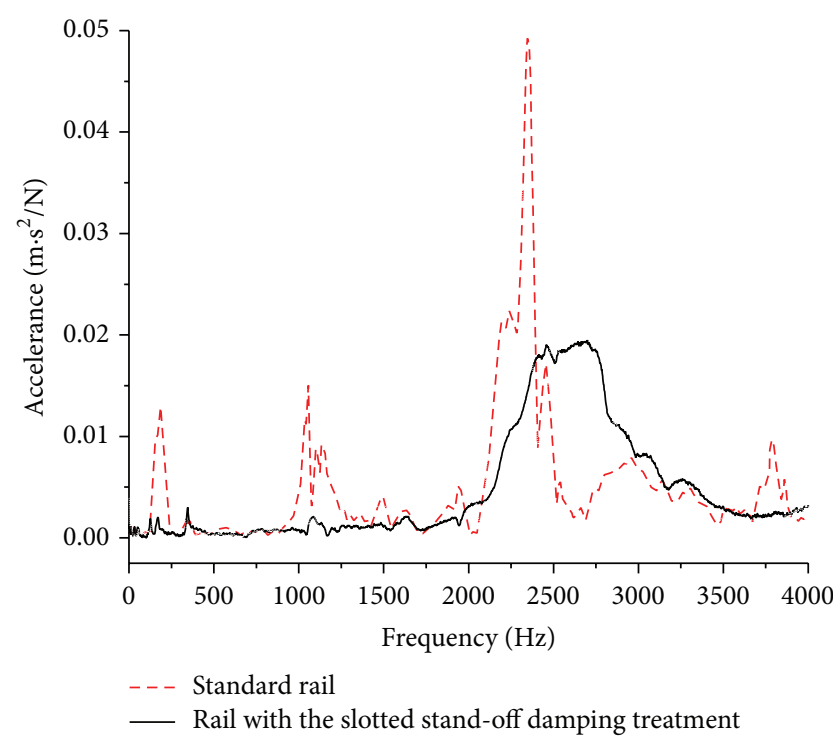

(b) Lateral accelerance of the rail head

FIGURE 12: Accelerance of the rail head.

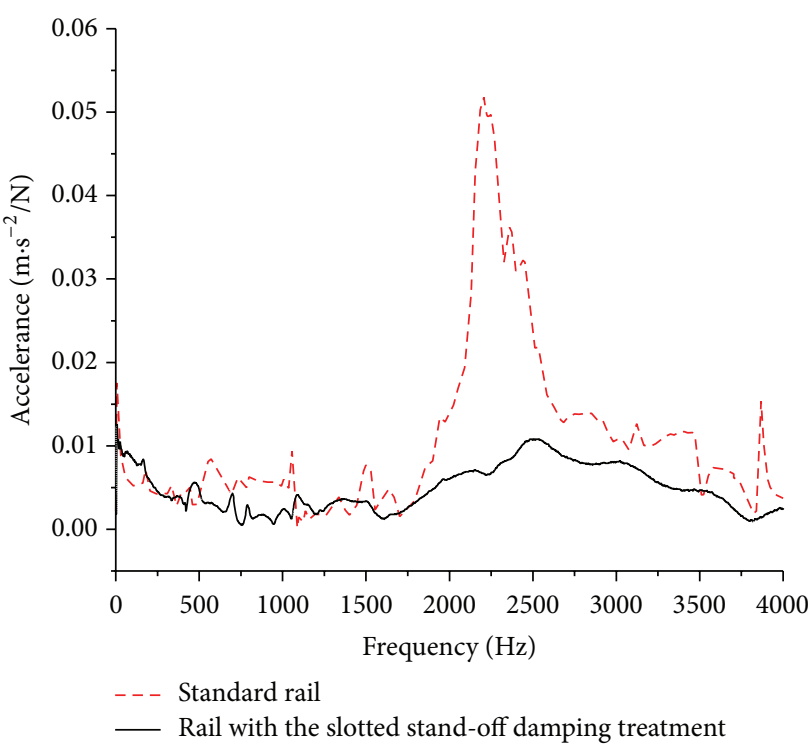

(a) Vertical accelerance of the rail waist

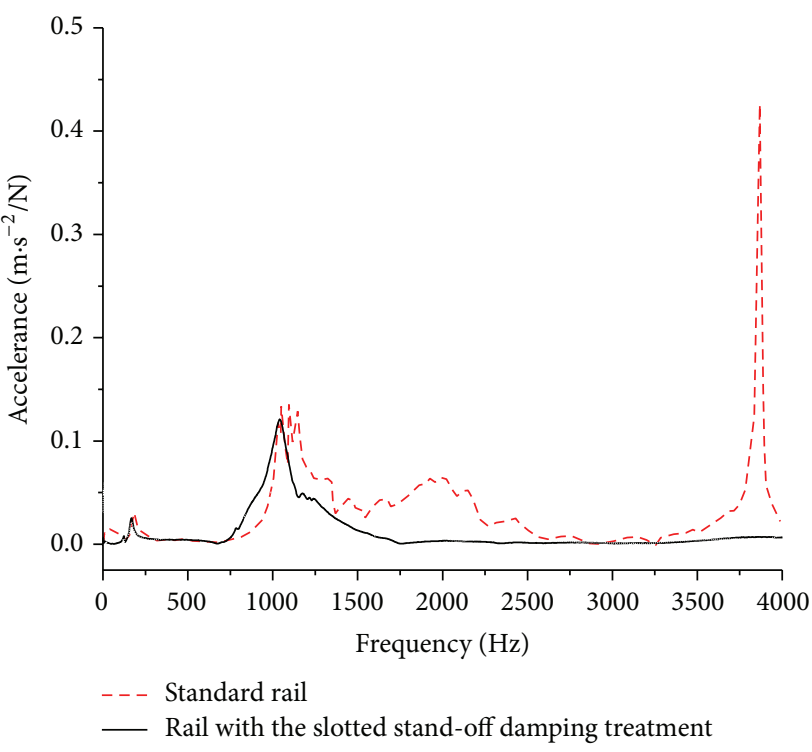

(b) Lateral accelerance of the rail waist

FIGURE 13: Accelerance of the rail waist.

length and $50 \mathrm{~cm}$ away from the rail centreline in the lateral direction, as shown in Figures 16 and 17.

The laboratory results for the rail with the slotted standoff damping treatment showed a significant reduction in sound emission compared to the standard rail under the vertical falling weight excitation, as shown in Figure 18; the radiation sound pressure level decreases by $8.2 \mathrm{~dB}$. It can also be observed from Figure 19 that the rail with the slotted standoff damping treatment produced less noise compared to the standard rail under the lateral falling weight excitation, with differences of up to $9.4 \mathrm{~dB}$. Furthermore, within the frequency range of $200-4000 \mathrm{~Hz}$, the noise level reduction is greater than that within the frequency range of $0-200 \mathrm{~Hz}$ under both vertical and lateral falling weight excitation.

\section{Conclusions}

The aim of this work was to design an innovative lownoise rail for high-speed trains. A slotted stand-off damping treatment was adopted for its effectiveness at reducing noise emission over a wide frequency range of $0-4000 \mathrm{~Hz}$ and for the possibility of applying it to existing rails. The choice of materials and treatment was the topic of the study. Several limitations had to be considered: ease of construction, 


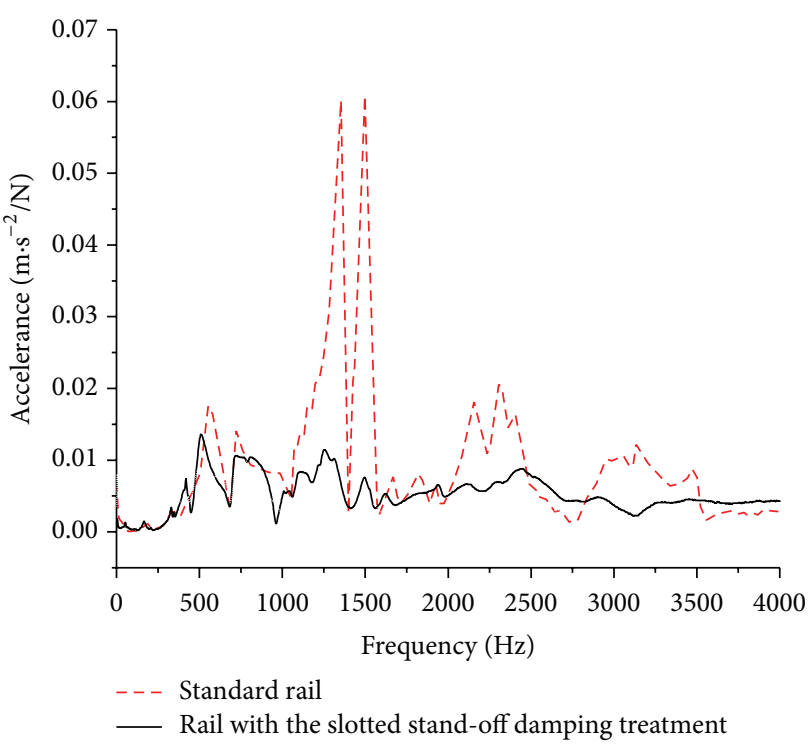

(a) Vertical accelerance of the rail foot

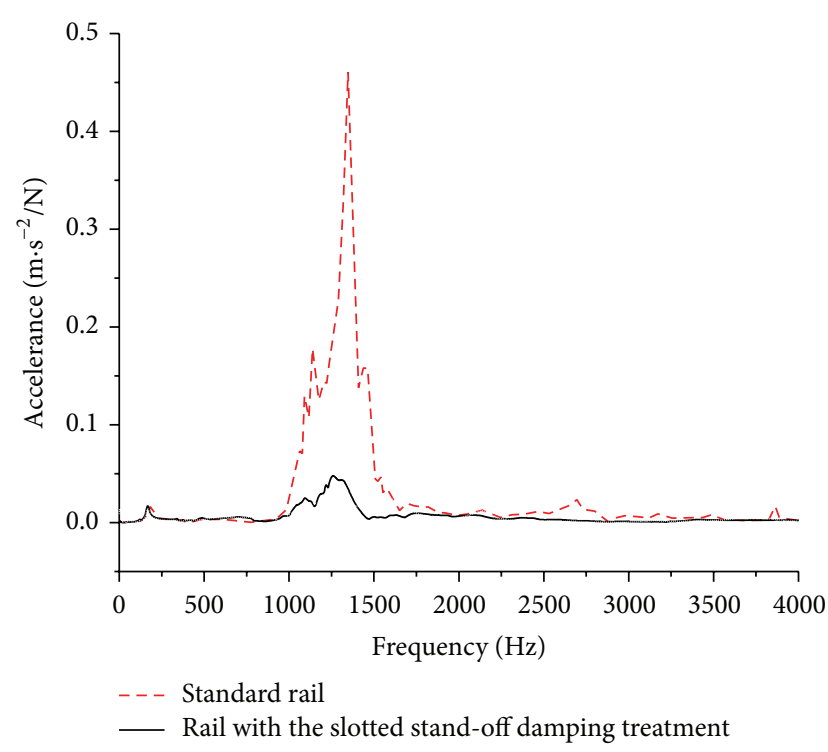

(b) Lateral accelerance of the rail foot

FIGURE 14: Accelerance of the rail foot.

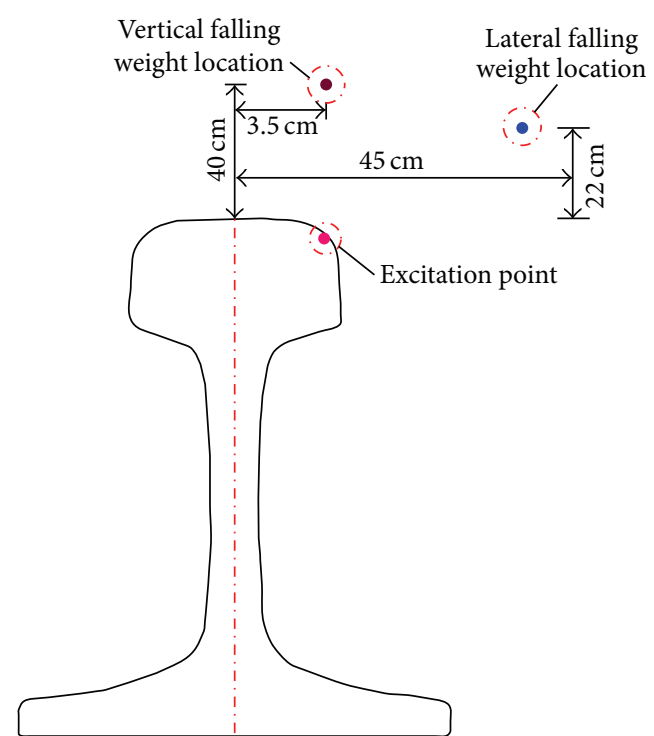

FIGURE 15: Location of the falling weight.

shaping and assembly, safety, commercial availability, weight, and costs. To better understand the influence of the design parameters and to aid in the selection of those parameters, a numerical finite element calculation method for the loss factor was developed, and extensive studies on an actual rail model were conducted. The results of these analyses led to the construction of a prototype with a $2 \mathrm{~mm}$ thick steel constraining layer, $6 \mathrm{~mm}$ thick Dyad606 stand-off layer with twelve slots that were $3 \mathrm{~mm}$ deep and $20 \mathrm{~mm}$ wide and were equally spaced at intervals of $30 \mathrm{~mm}$, and a viscoelastic layer of varying thickness according to the thickness of the rail

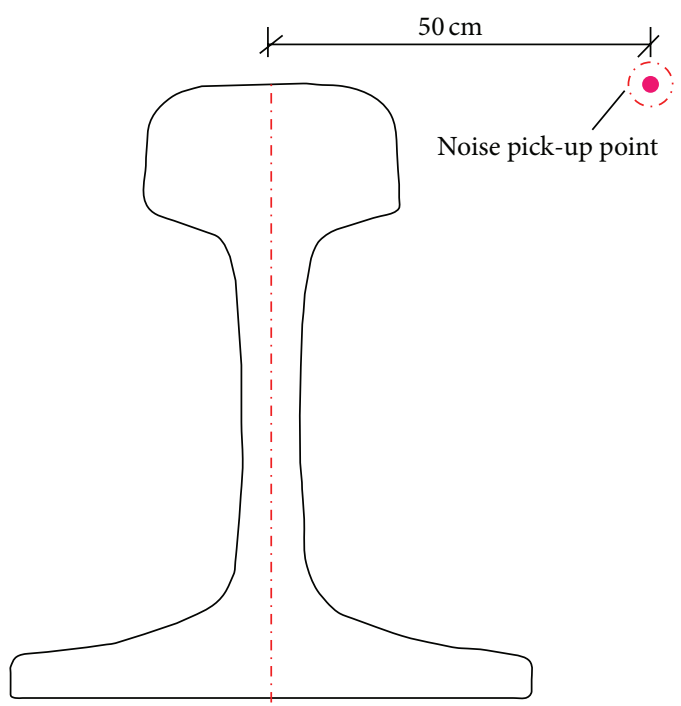

FIGURE 16: Location of the noise pick-up point.

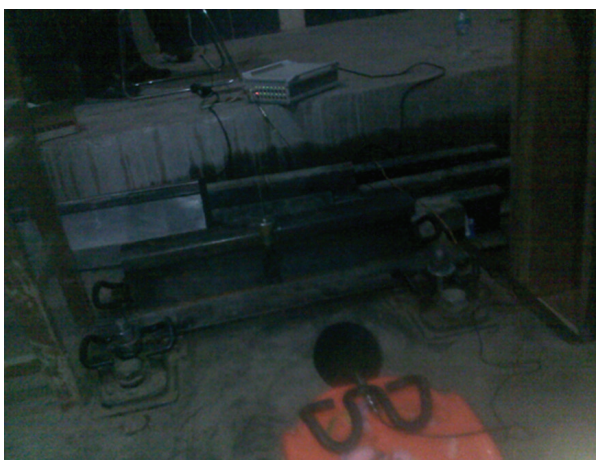

FIGURE 17: Laboratory vibroacoustic analysis. 


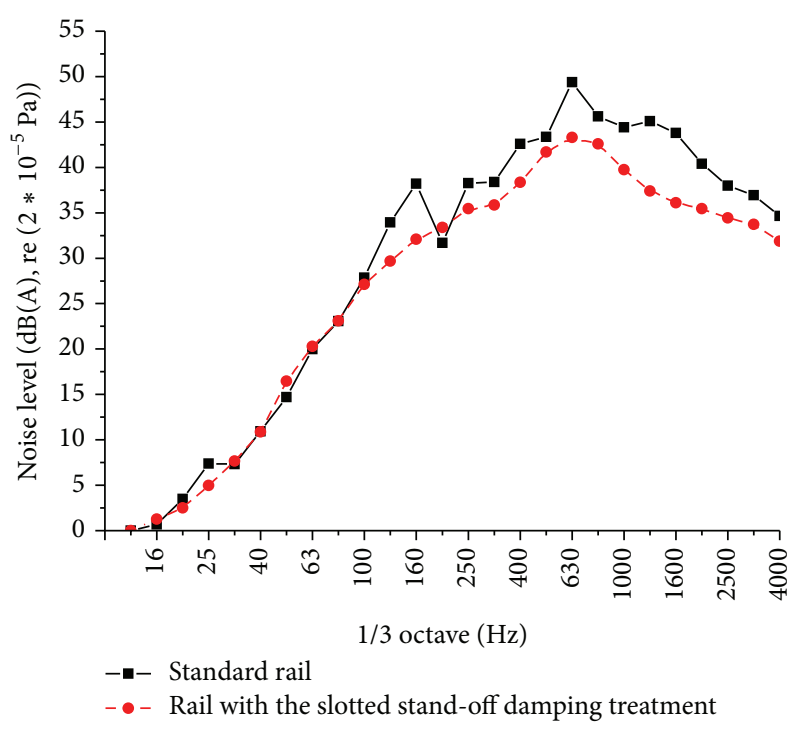

FIGURE 18: One-third octave curve in vertical excitation.

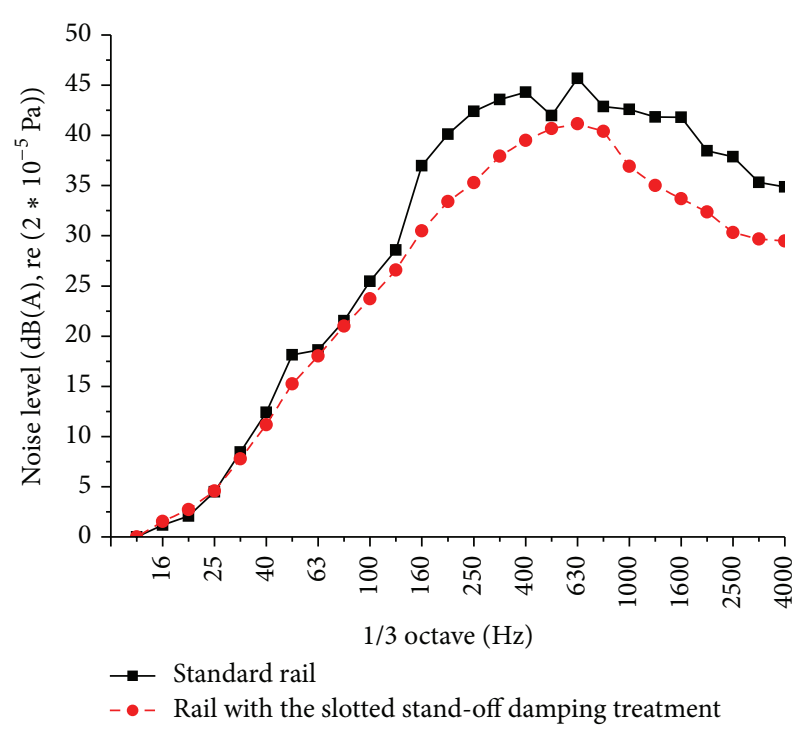

Figure 19: One-third octave curve in lateral excitation.

waist and rail foot, arranged on both sides of the rail in the zone under the rail head.

Laboratory measurements of the accelerance of the samples confirmed that the slotted stand-off damping treatment shows significant effects on decreasing the amplitude of the accelerance of the rail in the frequency range of $0-4000 \mathrm{~Hz}$, especially in the middle frequency range of $200-1000 \mathrm{~Hz}$ and the high frequency range of $1000-4000 \mathrm{~Hz}$. Noise emission measurements in the frequency range of major acoustic interest $(0-4000 \mathrm{~Hz})$ were also carried out in the laboratory. Compared to the standard rail emission, good noise reductions of approximately $8.2 \mathrm{~dB}$ under vertical excitation and $9.4 \mathrm{~dB}$ under lateral excitation were achieved in this test. In particular, the noise level reduction in the frequency range of $200-4000 \mathrm{~Hz}$ was greater than that within the frequency range of $0-200 \mathrm{~Hz}$ under both vertical and lateral falling weight excitation. Following these encouraging results, low-noise wheels proceeded to the production stage.

\section{Conflict of Interests}

The authors declare that there is no conflict of interests regarding the publication of this paper.

\section{Acknowledgment}

This research was sponsored by the National Natural Science Foundation of China, no. U1234201. This support is gratefully acknowledged. The results and opinions presented are those of the authors and do not necessarily reflect those of the sponsoring agencies.

\section{References}

[1] C. Talotte, P.-E. Gautier, D. J. Thompson, and C. Hanson, "Identification, modelling and reduction potential of railway noise sources: a critical survey," Journal of Sound and Vibration, vol. 267, no. 3, pp. 447-468, 2003.

[2] M. Szwarc, B. Kostek, J. Kotus, M. Szczodrak, and A. Czyzewski, "Problems of railway noise-a case study," The International Journal of Occupational Safety and Ergonomics, vol. 17, no. 3, pp. 309-325, 2011.

[3] J. J. Kalker and F. Périard, "Wheel-rail noise: impact, random, corrugation and tonal noise," Wear, vol. 191, no. 1-2, pp. 184-187, 1996.

[4] C. Collette, "Importance of the wheel vertical dynamics in the squeal noise mechanism on a scaled test bench," Shock and Vibration, vol. 19, no. 2, pp. 141-149, 2012.

[5] D. Thompson and C. Jones, "Noise and vibration from railway vehicles," in Handbook of Railway Vehicle Dynamics, pp. 211345, CRC Press, London, UK, 2006.

[6] D. T. Eadie, M. Santoro, and J. Kalousek, "Railway noise and the effect of top of rail liquid friction modifiers: changes in sound and vibration spectral distributions in curves," Wear, vol. 258, no. 7-8, pp. 1148-1155, 2005.

[7] D. Thompson, "Wheel-rail noise generation. Parts I to V," Journal of Sound and Vibration, vol. 161, no. 3, pp. 387-482, 1997.

[8] D. J. Thompson, "Experimental analysis of wave propagation in railway tracks," Journal of Sound and Vibration, vol. 203, no. 5, pp. 867-888, 1997.

[9] J. Maes and H. Sol, "A double tuned rail damper-increased damping at the two first pinned-pinned frequencies," Journal of Sound and Vibration, vol. 267, no. 3, pp. 721-737, 2003.

[10] W. Ho, B. Wong, and D. England, "Tuned mass damper for rail noise control," in Proceedings of the 10th International Workshop on Railway Noise, Noise and Vibration Mitigation for Rail Transportation Systems, Nagahama, Japan, 2010.

[11] F. Ltourneaux, F. Margiocchi, and F. Poisson, "Complete assessment of rail absorber performances on an operated track in France," in Proceedings of the World Congress on Railway Research, CDROM, Montreal, Canada, 2006.

[12] B. Asmussen, D. Stiebel, P. Kitson, D. Farrington, and D. Benton, "Reducing the noise emission by increasing the damping of the rail: results of a field test," Journal of Sound and Vibration, vol. 227, no. 1, pp. 711-721, 2005. 
[13] P.-B. Wei, H. Xia, Y.-M. Cao, and J.-W. Zhan, "Experimental study on vibration reduction of rail with compound damping board," Journal of Beijing Jiaotong University, vol. 31, no. 4, pp. 35-39, 2007 (Chinese).

[14] Z. W. Li, X. Y. Lei, and P. F. Zhang, "FEM analysis of damped rails for vibration reduction," Noise and Vibration Control, vol. 74, pp. 64-66, 2009 (Chinese).

[15] Y. I. N. Xue Jun, "Theoretical and experimental study on the labyrinth constrained damping layer," Noise and Vibration Control, vol. 58, pp. 148-152, 2007 (Chinese).

[16] P. Grootenhuis, "The control of vibrations with viscoelastic materials," Journal of Sound and Vibration, vol. 11, no. 4, pp. 421433, 1970.

[17] J. M. Yellin, I. Y. Shen, P. G. Reinhall, and P. Y. H. Huang, "An analytical and experimental analysis for a one-dimensional passive stand-off layer damping treatment," Journal of Vibration and Acoustics, vol. 122, no. 4, pp. 440-447, 2000.

[18] J. M. H. Yellin, I. Y. Shen, and P. G. Reinhall, "Experimental and finite element analysis of stand-off layer damping treatments for beams," in Proceedings of the ASME International Mechanical Engineering Congress and Exposition (IMECE '07), pp. 155-164, Seattle, Wash, USA, November 2007.

[19] W. Wei, "A model of rail track reacceptance analysis," Journal of Dalian Railway Institute, vol. 19, no. 4, pp. 33-38, 1998 (Chinese).

[20] International Organization for Standardization, "Vibration and shock-experimental determination of mechanical mobilitypart 5: measurements using impact excitation with an exciter which is not attached to the structure," Tech. Rep. ISO 7626-5, ISO, Geneva, Switzerland, 1994.

[21] International Organization for Standardization, "Acousticsdetermination of sound power levels and sound energy levels of noise sources using sound pressure-engineering methods for an essentially free field over a reflecting plane," ISO 3744-2010, 2010. 

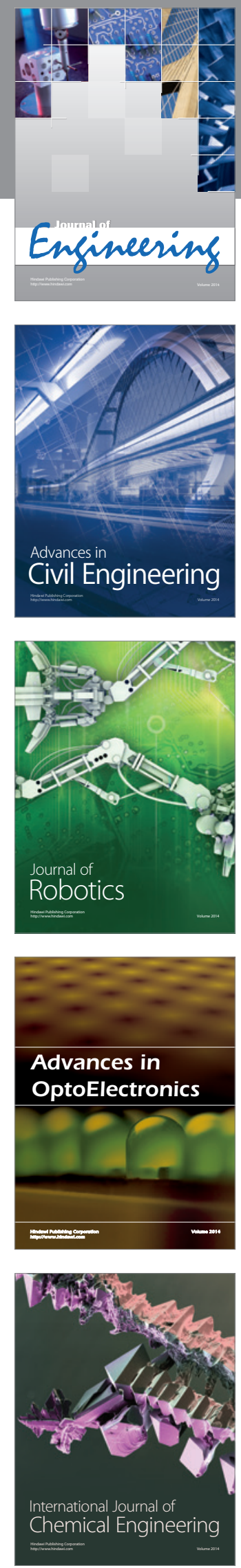

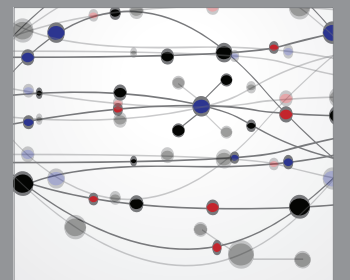

The Scientific World Journal
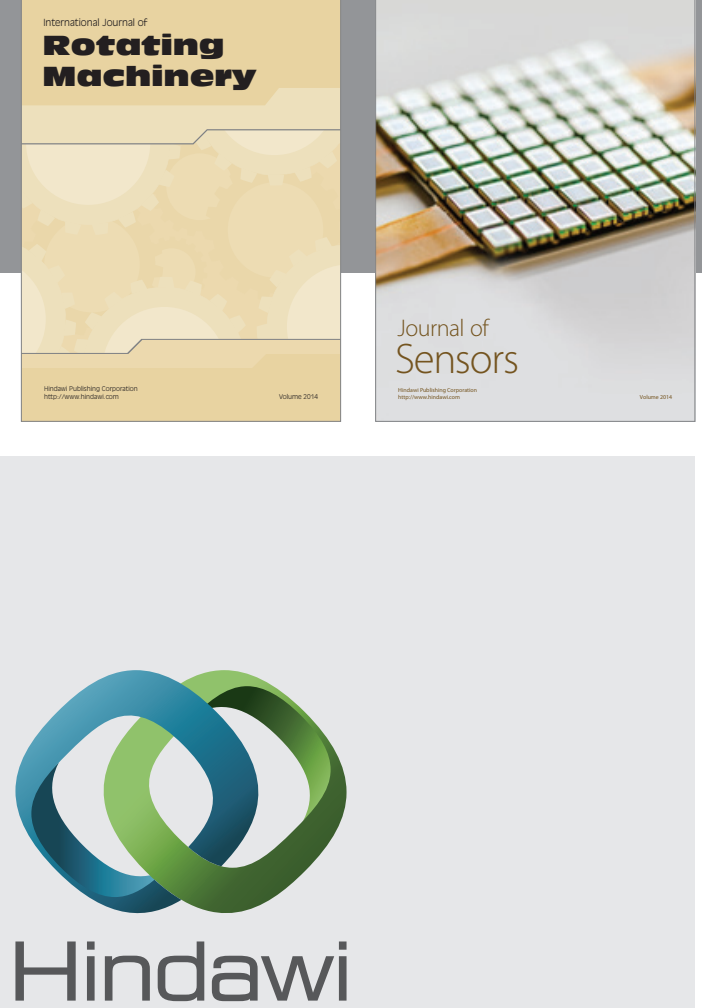

Submit your manuscripts at http://www.hindawi.com
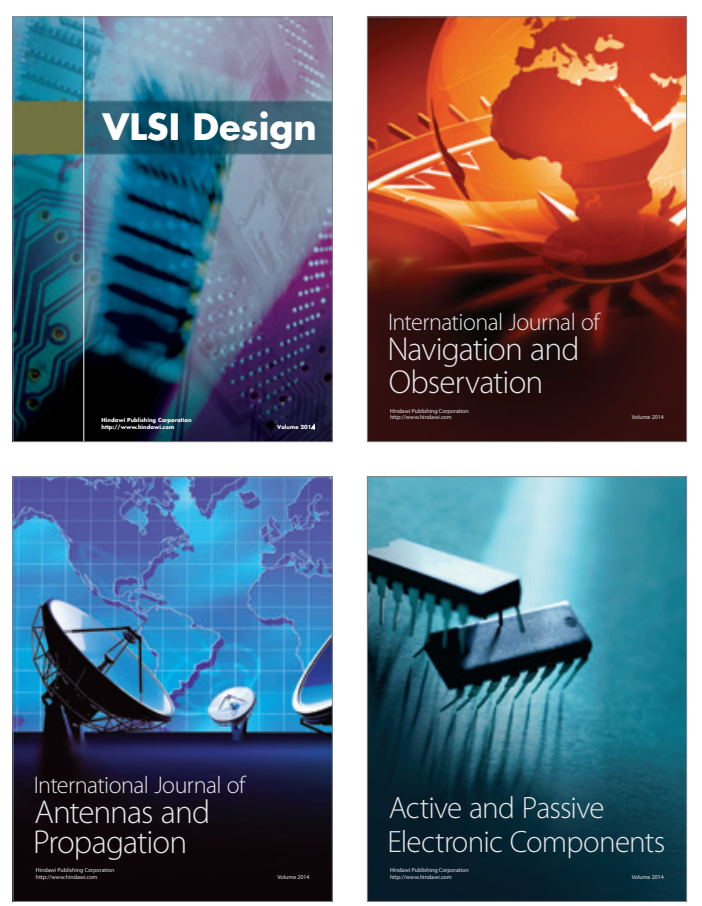
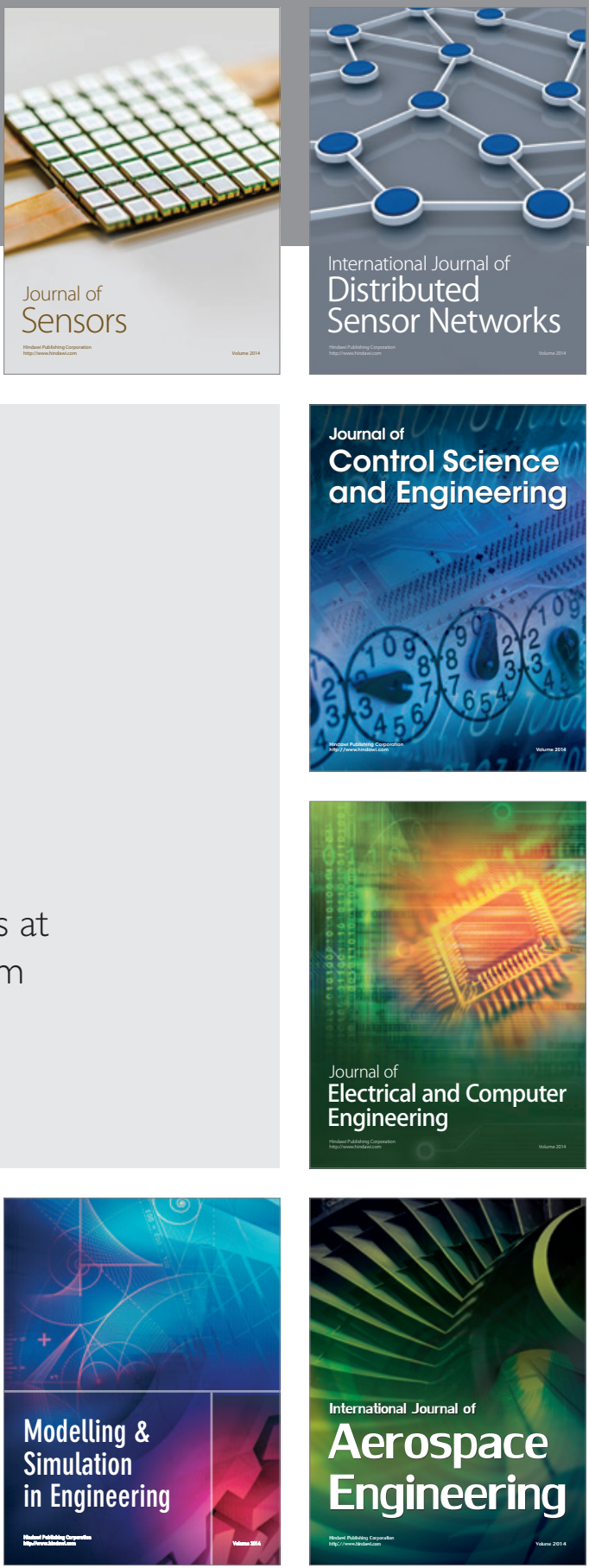

Journal of

Control Science

and Engineering
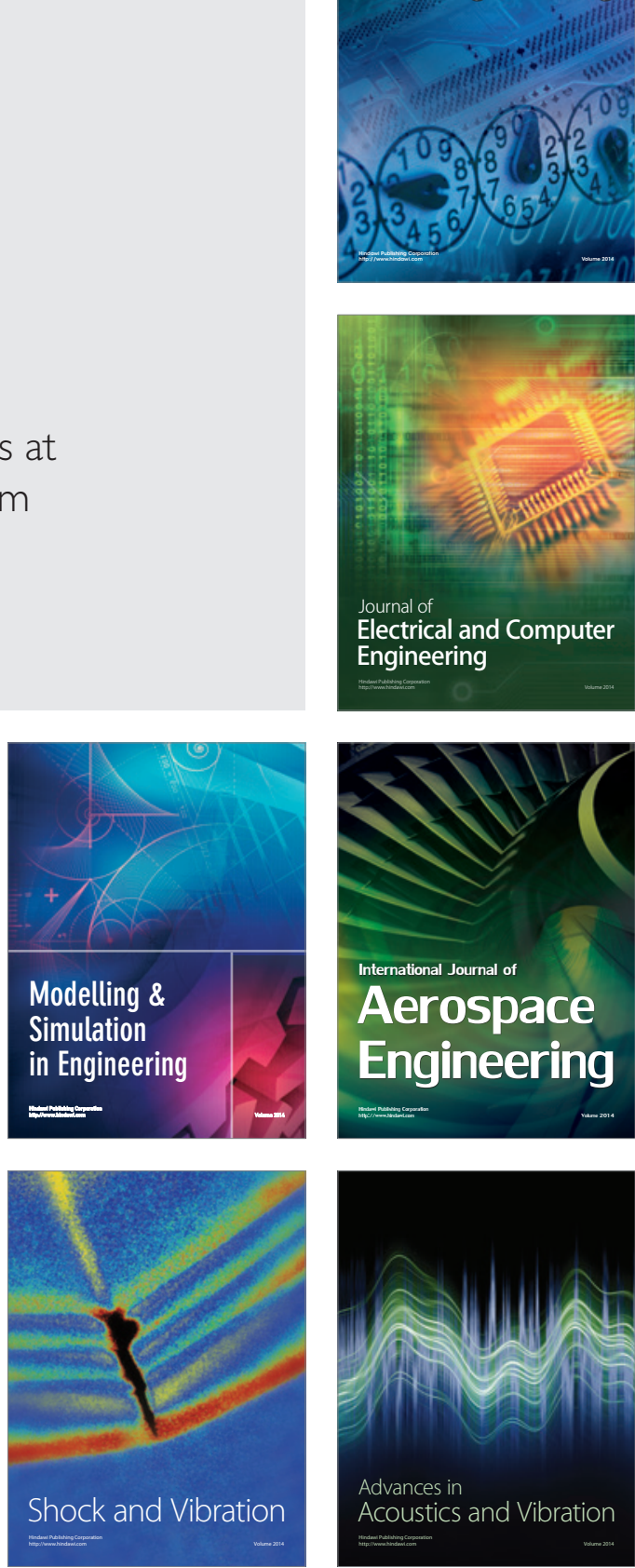